\title{
Equivalente esférico e valores da espessura da camada de fibras nervosas obtidas com o GDX ${ }^{T M}$ Scanning Laser System ${ }^{\circledR}$
}

\author{
Spherical equivalent and nerve fiber layer thickness assessed \\ with $G D X^{r M}$ Scanning Laser System ${ }^{\circledR}$
}

Lênio Souza Alvareng'a ${ }^{(1)}$ Christiane Rolim Moura ${ }^{(2)}$

Paulo Augusto de Arruda Mello ${ }^{(3)}$

João Antônio Prata Júnior (4)

\begin{tabular}{|c|}
\hline RESUMO \\
\hline 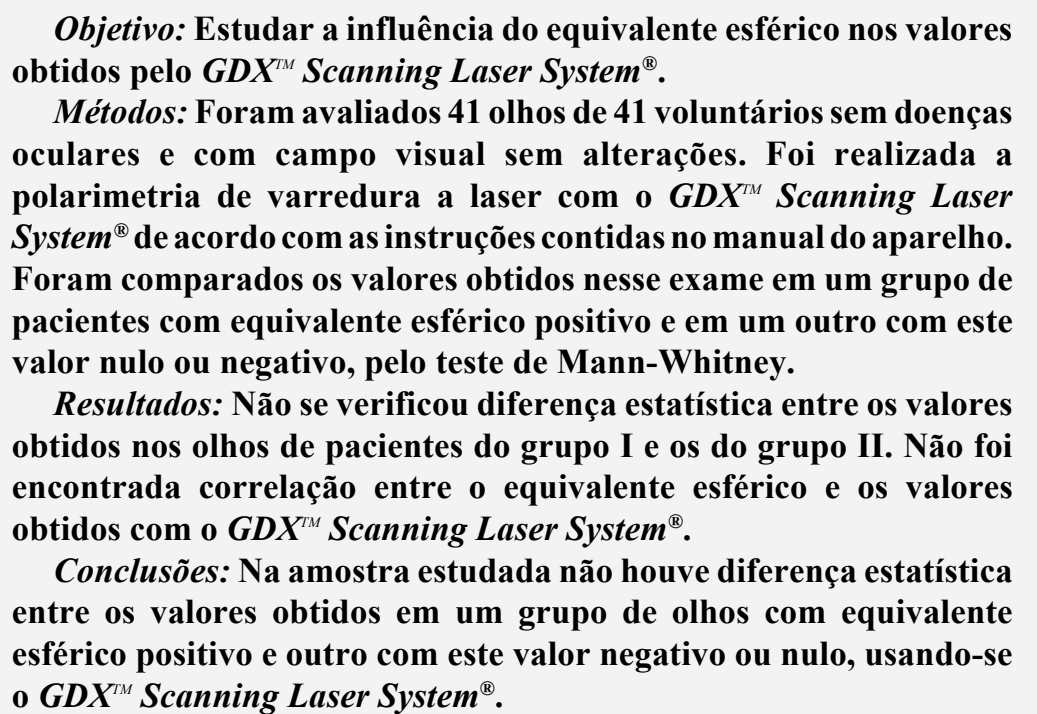 \\
\hline
\end{tabular}

Palavras-chave: Polarimetria; Camada de fibras nervosas; Equivalente esférico.

\section{INTRODUÇ̃̃̃O}

A polarimetria de varredura a laser (PVL) é utilizada para avaliar quantitativamente a camada de fibras nervosas da retina $(\mathrm{CFN})$ in vivo ${ }^{1-3}$. Vários estudos têm salientado a viabilidade do uso clínico deste método com valores de sensibilidade e especificidade satisfatórios para o diagnóstico de glaucoma ${ }^{4}$, ocorrendo pouca variabilidade entre medidas repetidas ${ }^{5-8}$. Estudos referem também uma associação entre as medidas tomadas com PVL e perda no campo visual de pacientes glaucomatosos. ${ }^{9,10}$.

O GDx Scanning Laser System, uma versão atualizada do polarímetro, fornece além das medidas da camada em seus vários pontos uma comparação destes resultados com um banco de dados, oferecendo parâmetros e índices. Esses valores são assinalados no resultado impresso como dentro ou fora da normalidade de acordo com a comparação com a database disponível ${ }^{11}$.

Para uma avaliação correta dos resultados obtidos com o $G D X^{T M}$ Scanning Laser System ${ }^{\circledR}$ faz-se necessário o conhecimento de outras variáveis que possam influenciar na aquisição destas medidas. Estudos salientam as influências da raça e da idade sobre os valores da espessura ${ }^{12,13}$. Entretanto, os efeitos de outros fatores, tais como as ametropias, ainda não 
foram estudados. Tal preocupação reside no fato de que a PVL baseia-se na birefringência da CFN para quantificar a sua espessura através da correlação com o atraso polarimétrico. Esse atraso é originado quando um feixe luminoso polarizado, que é emitido pelo aparelho percorre a $\mathrm{CFN}^{1-3}$. Ao passar pelo complexo óptico ocular bidirecionalmente esse feixe poderia sofrer a influência de ametropias.

O presente estudo visa analisar a influência de ametropias, através da análise do equivalente esférico, nas medidas obtidas e na análise estatística com o $G D X^{m}$ Scanning Laser System ${ }^{\circledR}$ num grupo de voluntários sem doenças oculares.

\section{PACIENTES E MÉTODOS}

Foram selecionados 41 voluntários com idade mínima de 40 anos e examinados no Setor de Glaucoma do Departamento de Oftalmologia da Universidade Federal de São Paulo (UNIFESP) / Escola Paulista de Medicina. Foram considerados critérios de inclusão: Exame biomicroscópico do segmento anterior e fundoscopia sem alterações, três medidas de pressão intra-ocular menores do que $21 \mathrm{mmHg}$, tomadas em ocasiões diferentes, disco óptico avaliado à biomicroscopia de fundo com relação escavação/disco menor ou igual a 0,5 no eixo vertical e sem alterações compatíveis com dano glaucomatoso. O campo visual central dos voluntários foi analisado com perimetria computadorizada (Humphrey Field Analyser 24-2 ou 30-2) e para inclusão todos apresentaram campo visual sem alterações. Foram excluídos aqueles com história de trauma ocular, cirurgia intra-ocular prévia ou acuidade visual menor ou igual a 0,3 . Não foram incluídos pacientes com erro refracional superior $\mathrm{a} \pm 6,00 \mathrm{DE}$.

Dados de identificação, sexo, raça, e idade foram registrados. Os indivíduos foram submetidos à refração dinâmica e divididos em dois grupos:

- Grupo I - Voluntários com equivalente esférico positivo

- Grupo II- Voluntários com equivalente esférico nulo ou negativo

Esses voluntários foram submetidos à PVL por meio do $G^{\prime w}$ Scanning Laser System ${ }^{\circledR}$. Foram considerados no estudo apenas os olhos direitos. O exame foi realizado sem cicloplegia e/ou midríase medicamentosa. Considerou-se apenas imagens de boa qualidade, classificadas como "pass" pelo programa do aparelho. A imagem adotada no estudo foi a média de três medidas. As razões e valores selecionados nesse estudo para a análise estatística foram aqueles fornecidos pelo $G D X^{i M}$ Scanning Laser System ${ }^{\circledR}$. Tais razões e valores foram: Simetria, Razão Superior, Razão Inferior, Superior/Nasal, Modulação Máxima, Modulação da Elipse, Média da Espessura, Média da Elipse, Média Superior, Média Inferior e Superior Integral. Todos os exames de análise da CFN bem como o exame oftalmológico, foram realizados pelo mesmo examinador, em sala com iluminação constante.

O teste de Mann-Whytney foi utilizado para se comparar os valores medianos das variáveis supracitadas entre os dois grupos e o teste de correlação de Spearman para avaliar a correlação entre as razões e valores obtidos em cada paciente com o seu equivalente esférico.

Em todos os casos o valor de significância para a rejeição da hipótese de nulidade foi fixado em um valor menor ou igual a $0,05(5 \%)$.

\section{RESULTADOS}

Estudou-se 41 olhos direitos de 41 indivíduos sem doenças oculares, totalizando $33(80,5 \%)$ do sexo feminino e 8 $(19,5 \%)$ do sexo masculino. Esta amostra compunha-se de : $27(65,8 \%)$ pacientes da raça branca, $13(31,7 \%)$ pardos e 1 $(2,5 \%)$ negro. A idade média foi de 59 anos. Não houve diferença estatística quanto a idade ou dados demográficos entre os grupos.

O equivalente esférico, obtido através de refração dinâmica da população estudada variou de $-2,75 \mathrm{DE}$ a $+4,75 \mathrm{DE}$ (média de + 0,57DE $\pm 1,55$ ) (Gráfico 1).

No Grupo I este valor variou entre $+0,12 \mathrm{DE}$ a $+4,75 \mathrm{DE}$ (Média de $+1,59 \pm 1,23 \mathrm{DE}$ e mediana de $+1,25 \mathrm{DE}$ ) enquanto no Grupo II observou-se uma variação desse valor de-2,75 DE a zero DE (Média de -0,65 + 0,79 DE e mediana de - 0,50 DE). O valor cilíndrico encontrado no Grupo I variou de zero $\mathrm{DC}$ a $-2.00 \mathrm{DC}$ (média de $0,75 \mathrm{DC} \pm 0,3$ ) e no Grupo II de zero DC a $-2,50$ DC (média de $1,1 \mathrm{DC} \pm 0,4$ ).

Os valores medianos dos índices e razões calculados pelo GDx Scanning Laser System, em cada grupo, foram comparados pelo teste de Mann-Whytney e não se verificou diferença significante (Tabela 1).

Através do método de Spearmann analisou-se o equivalente esférico com os índices e razões obtidos com o $G D X^{I M}$ Scanning Laser System ${ }^{\circledR}$ não sendo encontranda correlação estasticamente significante.

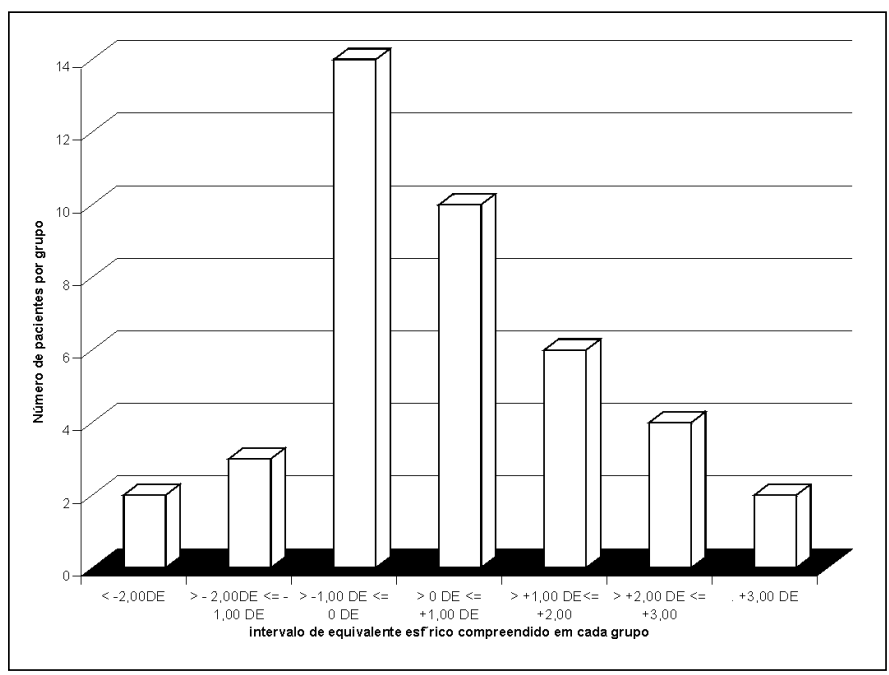

Gráfico 1. Valores de equivalente esférico na população estudada 


\begin{tabular}{|c|c|c|c|}
\hline & Grupo I * & Grupo II * & \\
\hline Simetria & 1,09 & 1,04 & N.S. \\
\hline Razão Superior & 2,57 & 2,75 & N.S. \\
\hline Razão Inferior & 2,55 & 2,47 & N.S. \\
\hline Superior/ Nasal & 1,87 & 1,99 & N.S. \\
\hline Modulação Máxima & 1,67 & 1,89 & N.S. \\
\hline Modulação da Elipse & 2,66 & 2,89 & N.S. \\
\hline Média da Espessura $(\mu \mathrm{m})$ & 68,00 & 68,00 & N.S. \\
\hline Média da Elipse $(\mu \mathrm{m})$ & 72,00 & 70,00 & N.S. \\
\hline Média Superior $(\mu \mathrm{m})$ & 83,00 & 77,00 & N.S. \\
\hline Média Inferior $(\mu \mathrm{m})$ & 81,00 & 84,00 & N.S. \\
\hline Superior Integral $(\mu \mathrm{m})$ & 0,23 & 0,22 & N.S. \\
\hline
\end{tabular}

Em nenhum caso, o exame da CFN mostrou-se fora da normalidade, segundo análise estatística do equipamento.

\section{DISCUSSÃO}

Como toda nova tecnologia, é fundamental o conhecimento de fatores que possam influenciar nos resultados obtidos. No caso da análise da CFN, poucos estudos encontram-se disponíveis com este objetivo. A motivação para a realização deste estudo deveu-se primariamente ao total desconhecimento da influência de ametropias sobre os resultados do exame, principalmente considerando que tal variável faz parte do conjunto de dados do paciente que são inseridos no programa. É interessante ressaltar, que quando esta variável é inserida no programa ocorre um erro de processamento de causa desconhecida.

Os resultados do presente estudo foram comparados em grupos homogêneos de indivíduos sem doenças oculares, a fim de dirimir possíveis influências de outras variáveis. Nestes, as ametropias detectadas não mostraram influência significativa sobre os valores da espessura da CFN quando obtida com o GDX $X^{T M}$ Scanning Laser System ${ }^{\circledR}$.

Este achado não é descrito na literatura e há a possibilidade de que se tenha decorrido da discreta diferença do equivalente esférico entre os dois grupos. Neste aspecto, cabe ressaltar que objetivou-se a análise exatamente dentro dos valores de equivalente esférico mais presentes na população ${ }^{14}$. Sem dúvida existe a possibilidade de que altas ametropias apresentem alguma influência nos resultados. Tal possibilidade encontra-se em estudo.

Como era esperado, não foram detectados exames considerados anormais pelo programa estatístico do equipamento nos indivíduos estudados. Tal fato pode ser considerado um ponto favorável à possibilidade de identificação de pacientes glaucomatosos com GDX $X^{T M}$ Scanning Laser System ${ }^{\circledR}$.

Os resultados do presente estudo não mostram influência de ametropias leves ou moderadas sobre os valores obtidos com o GDX $X^{T M}$ Scanning Laser System ${ }^{\circledR}$.

\section{SUMMARY}

Purpose: To evaluate the effect of spherical equivalent on the acquisition of nerve fiber layer (NFL) thickness with $\mathrm{GDX}^{\mathrm{TM}}$ Scanning Laser System ${ }^{\circledR}$.

Methods: Forty-one eyes of 41 volunteers were enrolled in this study. All of them presented with no ocular disease and no visual field defect. The NFL thickness was measured with GDX $^{\mathrm{TM}}$ Scanning Laser System ${ }^{\circledR}$ as described in its manual. The values obtained in a group of volunteers with negative spherical equivalent (group I) were compared to those from a group with a positive spherical equivalent (group II) by the Mann-Whitney test.

Results: There was no statistical difference between measurements in eyes of group I and those in group II. The NFL thickness measurements were not correlated with the spherical equivalent.

Conclusions: In the studied group there was no statistical difference in the $\mathrm{GDX}^{\mathrm{TM}}$ Scanning Laser System ${ }^{\circledR}$ parameters related to spherical equivalent.

Keywords: Polarimetry; Nerve fiber layer and Spherical equivalent.

\section{REFERÊNCIAS BIBLIOGRAFICAS}

1. Weinreb RN, Dreher AW, Coleman A, Quigley H, Shaw B, Reiter K. Histopatologic validation of Fourier-ellipsometry measurements of retinal nerve fiber layer thickness. Arch Ophthalmol 1990;108:557-60.

2. Dreher AW et al. Spatially resolved birefringence of the retinal nerve fiber assessed with a retinal laser ellipsometer. Applied Optics 1992;31(19):3730-5.

3. Dreher AW, Reiter K. Retinal laser ellipsometry: a new method for measuring the retinal nerve fiber layer thickness distribution? Clin Vision Sci 1992;481-8.

4. Galvão Filho, Suzanna Jr R. Estudo da camada de fibras nervosas em pacientes normais e glaucomatosos. Rev Bras Oftal 1999;58(1):15-8.

5. Duarte FB, Paranhos Jr, Galhardo RA et al. Reprodutibilidade do exame da medida da camada de fibras nervosas da retina por meio da polarimetria de varredura a laser. Arq Bras Oftal 1999;62(5).

6. Weinreb RN et al. Scanning laser polarimetry to measure the nerve fiber layer of the normal and glaucomatous eyes. Am J Ophthalmol 1995;119(5):627-36.

7. Tjon-Fo-Sang MJ, Lemij HG. The sensitivity and specificity of nerve fiber layer measurements in glaucoma as determined with scanning laser polarimetry. Am J Ophthalmol 1997;123:62-8.

8. Suzanna Jr R, Takahashi WI, Nakamura NFR. Sensibilidade e especificidade da avaliação da camada de fibras nervosas examinadas através da polarimetria de varredura a laser. Rev Bras Oftal 1998;57:17.

9. Anton A, Zangwill L, Emdadi A, Weinreb RN. Nerve fiber layer measurements with scanning laser polarimetry in ocular hypertension. Arch Ophthalmol 1997;115:331-4.

10. Weinreb RN et al. Association between quantitative nerve fiber layer measurement and visual field loss in glaucoma. Am J Ophthalmol 1995;120:732-8.

11. NFA ${ }^{\text {TM }}$ System Manual; Laser Diagnostic Technologies,Inc. San Diego, CA.

12. QI-MIN CHI et al. Evaluation of the effect of aging on the retinal nerve fiber layer thickness using scanning laser polarimetry. Journal of Glaucoma 1995;4:406-13.

13. Tjon-Fo-Sang MJ et al. Retinal nerve fiber layer measurements in normal black subjects as determined with scanning laser polarimetry. Ophthalmol 1998;105:78.

14. Meltzer DW et al. Optics, Refraction and contact lenses-Section 3 San Francisco: American Academy of Ophthalmology, 1996;150-3. 